\title{
The role of OCT4 immunohistochemistry in evaluation of retroperitoneal lymph node dissections: a pilot study
}

\author{
Muhammad T Idrees ${ }^{1}$, Sean R Williamson ${ }^{1}$, Theodore W Kieffer ${ }^{1}$ and Liang Cheng ${ }^{1,2}$ \\ ${ }^{1}$ Department of Pathology and Laboratory Medicine, Indiana University School of Medicine, Indianapolis, \\ IN, USA and ${ }^{2}$ Department of Urology, Indiana University School of Medicine, Indianapolis, IN, USA
}

\begin{abstract}
We investigated the role of OCT4 immunohistochemical staining in detecting germ cell tumor lymph node metastases. Retroperitoneal lymph node dissection is important for staging and treatment of testicular germ cell tumors, and OCT4 is sensitive and specific for pluripotent testicular germ cell tumors; however, micrometastases, particularly from seminoma, can be difficult to detect. We examined 262 lymph nodes in 45 retroperitoneal lymph node dissection specimens from germ cell tumor patients. Specimens were categorized as postchemotherapy and untreated retroperitoneal lymph node dissection with or without clinical suspicion, based on lymphadenopathy or elevated serum germ cell tumor markers. Sections were stained with anti-OCT4 antibody. Twenty-one additional positive lymph nodes in 12 cases were detected to harbor scattered seminoma cells, singly and in small clusters, from 256 previously considered benign in: untreated retroperitoneal lymph node dissection with clinical suspicion (13\% increase), postchemotherapy retroperitoneal lymph node dissection (7\%), and untreated retroperitoneal lymph node dissection without suspicion (4\%). However, no patient with an entirely negative dissection specimen was reclassified as positive. OCT4 immunohistochemistry detected scattered seminoma cells and small clusters of seminoma cells in lymph nodes previously considered to be benign for an overall increase of $8 \%$, greatest in the setting of untreated retroperitoneal lymph node dissection with clinical suspicion. However, immunohistochemistry did not convert any entirely negative specimen to positive. Future studies will be useful to determine whether the small volume of disease detected by immunohistochemistry has the same impact as routinely detected lymph node metastases.
\end{abstract}

Modern Pathology (2013) 26, 1613-1619; doi:10.1038/modpathol.2013.110; published online 14 June 2013

Keywords: germ cell tumors; immunohistochemistry; lymph node dissection; micrometastases; OCT4; seminoma

OCT4 (OCT3/4, POU5F1) is a POU-domain, octamer-binding transcription factor that is involved with regulation of downstream targets, such as NANOG, which are involved with maintaining the pluripotency of embryonic stem cells, adult stem cells, and germ cells. ${ }^{1-8}$ Disruption of the OCT4 gene, located on chromosome $6 \mathrm{p} 21.3$, has been shown to be essential for the self-renewal of embryonic stem cells in mouse development. ${ }^{9}$

It has been demonstrated that OCT4 is expressed in testicular germ cell tumors and that immunostaining with antibody to OCT4 is a useful diag-

Correspondence: Dr MT Idrees, MD, Department of Pathology and Laboratory Medicine, Indiana University School of Medicine, 350 West 11th Street, 4th Floor, Indianapolis, IN 46202, USA.

E-mail: midrees@iupui.edu

Received 19 November 2012; revised 21 February 2013; accepted 17 May 2013; published online 14 June 2013 nostic tool in the identification of primary testicular embryonal carcinomas and seminomas. ${ }^{1}$ It has also been shown to be a sensitive marker for testicular intratubular germ cell neoplasia. ${ }^{2}$ OCT4 staining is routinely used in the diagnosis of primary germ cell tumors of the testis and other sites, ${ }^{10}$ as well for confirmation of metastatic germ cell tumor. However, the use of OCT4 in the detection of occult nodal metastasis, similar to the use of melanocytic or cytokeratin markers in the identification of melanoma or breast cancer cells in sentinel lymph nodes, has not been investigated and applied in the evaluation of retroperitoneal lymph node dissection specimens. We undertook immunohistochemical staining for OCT4 in retroperitoneal lymph node dissection specimens to assess the utility of this marker as a diagnostic tool for occult lymph node metastasis in three clinical scenarios. 


\section{Materials and methods}

An electronic database search was performed for retroperitoneal lymph node dissection specimens in the Indiana University Health surgical pathology records between the years of 1995 and 2010 . As seminoma, in particular, exhibits a tendency to infiltrate as individual cells or small clusters of cells, retroperitoneal lymph node dissection specimens were included in the study if the patient had a known primary or metastatic germ cell tumor with a seminomatous component and the retroperitoneal lymph node dissection specimen included negative lymph nodes. Electronic medical records were reviewed to identify patients who received chemotherapy before the lymph node dissection and whether the retroperitoneal lymph node dissection was performed based on clinical suspicion for metastatic disease. Clinical suspicion was defined as enlarging lymph nodes, a mass detected by imaging, or elevation of serum markers. Of the cases available, 45 met criteria for inclusion and were divided into three groups with distinct clinical scenarios. All 21 retroperitoneal lymph node dissections that were performed after chemotherapy (postchemotherapy retroperitoneal lymph node dissection) were done as a result of clinical suspicion for metastasis. Of the retroperitoneal lymph node dissections that did not follow adjuvant chemotherapy (untreated retroperitoneal lymph node dissection), 13 were performed with clinical suspicion for metastasis while 11 were elective or recommended.

As part of the routine surgical pathology practice during the study period, lymph nodes without grossly visible tumor were submitted entirely for microscopic examination, individually or with multiple lymph nodes in the same histology cassette, and a single hematoxylin and eosin (H\&E)-stained section was prepared from each block. The original sections were retrospectively reviewed and tissue blocks were selected for inclusion in the study if they contained one or more lymph nodes without light microscopically identifiable tumor. Tissue blocks containing lymph nodes partially or entirely replaced by either necrotic tumor or fibrosis, hemosiderin deposition, and xanthomatous reaction indicative of treatment effect on tumor were excluded from study due to the possibility of retained immunohistochemical reactivity in nonviable cells. For positive internal control, slides that contained a light microscopically positive lymph node in addition to the negative lymph nodes were chosen when possible. Fourmicrometer-thick serial sections were prepared from the selected paraffin blocks. Two consecutive sections were stained with $\mathrm{H} \& \mathrm{E}$ and immunohistochemistry. Immunohistochemical staining was performed on an automated immunostainer (Dako, Carpinteria, CA, USA) with a polyclonal goat antiOCT4 antibody (C20, sc 8629; Santa Cruz Biotechnology, Santa Cruz, CA, USA; 1:500 dilution, $30 \mathrm{~min}$ at room temperature) directed against the $-\mathrm{COOH}$ terminus of the protein. Antigen retrieval was carried out by heating sections in $1 \mathrm{mmol} / \mathrm{l}$ ethylene diamine tetra acetic acid ( $\mathrm{pH} 8.0$ ) for $30 \mathrm{~min}$. Endogenous peroxidase activity was inactivated by incubation in $3 \% \mathrm{H}_{2} \mathrm{O}_{2}$ for $15 \mathrm{~min}$. Nonspecificbinding sites were blocked using Protein Block (Dako) for $20 \mathrm{~min}$. As OCT4 is a nuclear transcription factor involved in gene regulation, only nuclear staining was considered a positive result. The H\&Estained slides were reviewed for any previously undetected tumor foci by light microscopy and subsequently compared with immunohistochemistry-stained slides.

\section{Results}

Results are summarized in Table 1. The 45 cases were divided as follows: 13 untreated retroperitoneal lymph node dissection (with clinical suspicion), 11 untreated retroperitoneal lymph node dissection (elective or recommended), and 21 postchemotherapy retroperitoneal lymph node dissection. In total, $34(76 \%)$ of the 45 cases were originally interpreted as positive for malignancy. We evaluated 262 lymph nodes in total, including 256 light microscopically negative lymph nodes.

Table 1 Positive lymph nodes before and after immunohistochemical staining with anti-OCT4 antibody

\begin{tabular}{|c|c|c|c|}
\hline Clinical scenario & $\begin{array}{c}\text { Positive lymph } \\
\text { nodes/total (original } \\
\text { light microscopy) }\end{array}$ & $\begin{array}{l}\text { New positive lymph } \\
\text { nodes/negative lymph } \\
\text { nodes studied }\end{array}$ & $\begin{array}{l}\text { Percentage } \\
\text { of change }^{\mathrm{a}}\end{array}$ \\
\hline Untreated retroperitoneal lymph node dissection with clinical suspicion & $26 / 227$ & $10 / 75$ & $13 \%$ \\
\hline Untreated retroperitoneal lymph node dissection without clinical suspicion & $8 / 237$ & $3 / 74$ & $4 \%$ \\
\hline $\begin{array}{l}\text { Postchemotherapy retroperitoneal lymph node dissection (clinically evident } \\
\text { disease) }\end{array}$ & $53 / 363$ & $8 / 107$ & $7 \%$ \\
\hline Total & $87 / 827$ & $21 / 256$ & $8 \%$ \\
\hline
\end{tabular}

Eighty-seven of 827 lymph nodes were originally diagnosed as containing metastatic disease based on original light microscopy. Of these, 256 negative lymph nodes were selected for OCT4 immunohistochemistry. No specimen that was originally considered to be entirely free of metastatic disease became positive after OCT4 staining; however, additional positive lymph nodes were identified in all of the three studied clinical scenarios, greatest in untreated retroperitoneal lymph node dissection with clinical suspicion for metastasis.

apercentage of change is based on new positive lymph nodes out of total negative lymph nodes subjected to immunohistochemistry. 
Six light microscopically positive lymph nodes were present in the same sections and were also examined as positive controls. For most patients, the testicular primary tumor was a mixed germ cell tumor (28 patients), of which 26 had a recognized seminoma component and 2 did not. In eight patients, only seminoma was identified in the original orchiectomy specimen. Five patients were considered to have a regressed or 'burnt out' testicular primary tumor, and the characteristics of the primary tumor were unknown in three patients. One patient had exclusively teratoma in the primary tumor, though metastases contained seminoma. Metastases in 34 patients consisted of seminoma, while 8 patients had other metastatic elements, including yolk sac tumor, embryonal carcinoma, and teratoma.

\section{Untreated Retroperitoneal Lymph Node Dissections with Clinical Suspicion for Metastasis}

Eleven (85\%) of the 13 untreated retroperitoneal lymph node dissections (with clinical suspicion) were originally interpreted as containing metastatic disease, while two were considered free of malignancy (total 26/227 lymph nodes). From these 13 cases, 30 slides were selected containing 79 lymph nodes. Four lymph nodes selected for staining were originally interpreted as positive for malignancy by light microscopy while the remaining 75 were free of disease. After OCT4 immunohistochemical staining, 10 additional positive lymph nodes were identified in 6 different cases from the 75 previously negative lymph nodes studied (13\% change). No positive lymph nodes were identified in cases originally considered to be entirely free of metastatic disease.

\section{Untreated Retroperitoneal Lymph Node Dissections without Clinical Suspicion for Metastasis}

Five $(45 \%)$ of the 11 untreated retroperitoneal lymph node dissections (elective or recommended) were originally interpreted as positive for metastatic disease, while 6 were free of malignancy (total 8/237 lymph nodes). From these 11 cases, 23 slides were selected containing 74 lymph nodes, all of which were originally considered to be free of disease. After OCT4 immunohistochemical staining, 3 additional positive lymph nodes were identified in 2 different cases totaling $3(4 \%)$ positive of the 74 previously negative lymph nodes studied (4\% change). No positive lymph nodes were identified in cases originally considered to be entirely free of metastatic disease.

\section{Postchemotherapy Retroperitoneal Lymph Node Dissections}

Eighteen $(86 \%)$ of the 21 postchemotherapy retroperitoneal lymph node dissections were originally interpreted as positive for metastatic disease, while 3 were considered to be free of malignancy (total 53/363 lymph nodes). From these 21 cases, 46 slides were selected containing 109 lymph nodes. Two of the lymph nodes selected for staining were originally considered to be positive for malignancy, while 107 were interpreted to be free of disease. After OCT4 immunohistochemical staining, 8 additional positive lymph nodes were identified in 4 different cases from the 107 previously negative lymph nodes studied ( $7 \%$ change).

\section{Total Number of Positive Lymph Nodes}

The total number of positive lymph nodes in the slides selected for staining increased from $6(2 \%)$ before OCT4 staining to $27(10 \%)$ after OCT4 staining out of the 262 total lymph nodes, yielding an additional 21 positive lymph nodes detected from the 256 originally considered benign (8\% increase). However, OCT4 staining did not identify malignant cells in cases that were originally considered to be free of disease. Hence, 11 (85\%) of the 13 untreated retroperitoneal lymph node dissection (with clinical suspicion), 5 (45\%) of the 11 untreated retroperitoneal lymph node dissection (elective or recommended), and $18(86 \%)$ of the 21 postchemotherapy retroperitoneal lymph node dissection contained metastatic disease both before and after OCT4 staining. The light microscopically positive lymph nodes that were also present in the OCT4-stained slides showed strong positive labeling in the tumor deposits as internal controls (Figures 1a and b). No previously undetected foci of metastatic tumor were identified by $\mathrm{H} \& \mathrm{E}$ staining alone. New lymph node metastases identified by OCT4 immunohistochemical staining consisted of infiltrating individual tumor cells and small clusters of tumor cells with round-to-squared nuclear contours and moderate amounts of cytoplasm, interpreted as seminoma cells. In some cases, retrospective examination of the same areas on the H\&E-stained slides demonstrated individual cells with enlarged, hyperchromatic nuclei, clumped nuclear chromatin, and prominent nucleoli within the subcapsular sinus or within the lymph node parenchyma admixed with sinus histiocytes (Figures 1c and d). In other cases, retrospective examination of the same areas on the H\&E-stained slide could not differentiate unequivocal tumor cells from sinus histiocytes or lymphoid cells (Figures 1e and f). OCT4 immunohistochemistry did not identify any foci of metastatic embryonal carcinoma, composed of more cohesive clusters of cells with increased nuclear pleomorphism, multiple prominent nucleoli, or irregular nuclear contours.

\section{Discussion}

In 2012, testicular cancer is estimated to account for 360 of the 301820 cancer-related deaths and 8590 of 

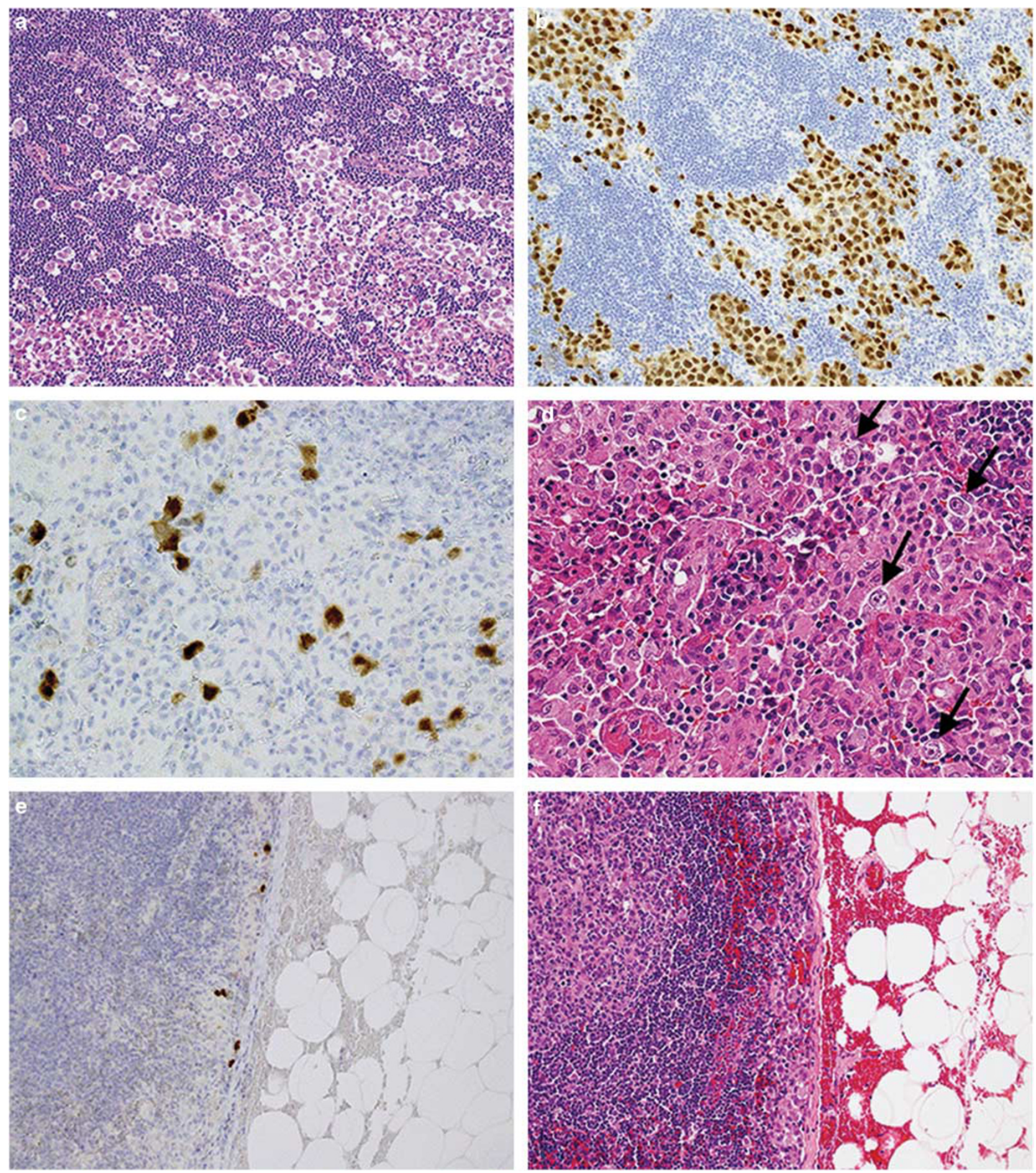

Figure 1 (a) Metastatic seminoma in a retroperitoneal lymph node, recognizable by light microscopy and (b) showing strongly positive nuclear labeling with anti-OCT4 antibody. (c) Another retroperitoneal lymph node contained occult metastasis of seminoma, evident by OCT4 immunohistochemistry showing small clusters and single seminoma cells admixed with sinus histiocytes. (d) The same field on the corresponding H\&E-stained section demonstrates scattered suspicious cells with prominent nucleoli (arrows), although fewer in number than those highlighted by immunohistochemistry. (e) Another lymph node shows scattered OCT4-positive cells in the subcapsular sinus that were not discernible by light microscopy (f), perhaps due to intermingling with sinus histiocytes or exposure upon cutting additional sections for immunohistochemistry.

the 848170 cancer diagnoses in men. ${ }^{11}$ Although less common than prostate or lung cancer $(241740$ diagnoses with 28170 deaths and 116470 diagnoses with 87750 deaths, respectively), ${ }^{11}$ the diagnosis of testicular cancer is particularly devastating as it often affects young men. Interestingly, cases of 
testicular cancer are steadily increasing without a known etiology, indicating that testicular cancer may be a more prevalent problem in years to come.

Patients with clinical stage I testicular germ cell tumors may choose surveillance, adjuvant therapy, or retroperitoneal lymph node dissection postorchiectomy to manage occult metastasis. Retroperitoneal lymph node dissection has staging and therapeutic value, making it a practical procedure in testicular germ cell tumors; however, proper staging can be difficult, particularly as seminoma exhibits a tendency to infiltrate as single cells and micrometastases sometimes occur.

OCT4 is used in many surgical pathology practices as a specific and sensitive marker for determining the subtype of primary or metastatic germ cell tumor; however, the usefulness of this antibody has not been evaluated in the detection of occult metastasis in retroperitoneal lymph node dissection. The use of specific markers in diagnosing occult metastasis has been utilized in a number of malignancies, such as breast carcinoma, melanoma, gynecologic malignancies, oral and head and neck malignancies, colorectal cancer, and Merkel cell carcinoma. ${ }^{12-17}$ Although originally developed in other organs, sentinel lymph node biopsy has gained widespread use in breast cancer. ${ }^{18}$ On the basis of the results of large randomized trials confirming its efficacy and reduced morbidity, sentinel lymph node biopsy has become a standard of care for axillary evaluation in breast cancer patients with early stage disease in many institutions. ${ }^{19-22}$ As such, a number of methodologies have been investigated to improve diagnostic yield in detecting lymph node metastases. In one such study, occult metastases were detected in $4 \%$ of breast sentinel lymph nodes previously reported to be benign, using a combination of deeper sectioning of the tissue blocks and cytokeratin immunohistochemistry. Overall case conversion rate was $10 \% .{ }^{23}$ Other studies have reported increased detection of occult metastases by similar methods, ranging from $9 \%$ to $33 \% .{ }^{24-27}$ Several markers, including cytokeratin AE1/AE3, pancytokeratin, CAM5.2, MAK-6, epithelial membrane antigen, MUC1, and cytokeratin 19, have been utilized in immunohistochemical evaluation of axillary sentinel lymph nodes. ${ }^{28-32}$ Likewise, new rapid cytokeratin immunohistochemistry techniques have recently emerged that have been reported to accurately assess the sentinel lymph node intraoperatively more quickly than previous methods. ${ }^{33-36}$ Additionally, multiple molecular markers, including hMAM-A and MAGE-A3, have been successfully used for detection of micrometastasis in breast sentinel lymph nodes. ${ }^{37}$ However, the impact of these findings on clinical outcome is debatable, and it has been suggested that the clinical benefit of such additional evaluation, ${ }^{18}$ including immunohistochemistry, in some cases may be limited..$^{23,24}$
Similar to these other organs, immunohistochemical evaluation of lymph nodes for occult metastases may be of value in directing prognosis and therapy for germ cell tumor patients who undergo retroperitoneal lymph node dissection, particularly if cases are categorized according to clinical suspicion. In this study, we identified additional positive lymph nodes using OCT4 immunohistochemistry that were previously interpreted as negative by light microscopy, although only in cases in which metastases had already been diagnosed by light microscopy in other lymph nodes. Interestingly, additional positive lymph nodes were identified in all the three clinical scenarios: postchemotherapy and untreated (untreated) with and without clinical suspicion. The overall additional volume of metastatic seminoma observed with OCT4 immunostaining was uniformly very small, consisting of single cells or small clusters of $<10$ cells. The additional metastatic foci were not $>1 \mathrm{~mm}$ in any case. The most common pattern consisted of dispersed single cells within the lymph node parenchyma admixed with sinus histiocytes. It is also of note that several of the cases showed single cells in the middle of the nodal parenchyma without involvement of subcapsular sinuses. In many cases, the metastatic tumor cells could not be easily identified on retrospective review of the H\&E-stained slides, possibly because the tumor cells were singly dispersed among sinus histiocytes or were not present on the original levels and were only exposed by the additional section cut for OCT4 immunostaining. It has also been recently noted that paraganglion cells demonstrate cytoplasmic staining with antibody to OCT4. ${ }^{38}$ Therefore, caution must be exercised to avoid interpretation of extranodal cells with cytoplasmic positivity for OCT4 as metastatic seminoma.

Cases were selected that had a seminomatous component in either the primary tumor or metastases, as seminoma, in particular, demonstrates a tendency to infiltrate as single noncohesive cells that may be easily overlooked; however, it is important to keep in mind that germ cell tumor metastases may also harbor components dissimilar from those of the primary tumor, at least partly attributable to transformation between germ cell tumor types before and after metastasis. ${ }^{39}$ In our experience, embryonal carcinoma typically forms larger, more cohesive aggregates of cells in lymph node metastases; however, small foci may sometimes resemble germinal centers at low magnification. OCT4 immunohistochemical antibody also labels embryonal carcinoma; however, we did not identify previously undetected foci of metastatic embryonal carcinoma in this study.

Overall, an $8 \%$ increase in the number of positive lymph nodes was detected using OCT4 immunostaining, although the number of positive cases remained the same after application of immunohistochemistry. These findings suggest that OCT4 
is a useful marker to identify micrometastasis in testicular germ cell tumor retroperitoneal lymph node dissection specimens, although these cases typically also have larger, light microscopically identifiable foci of metastatic disease. The risk of relapse after retroperitoneal lymph node dissection is generally thought to depend on the volume of metastatic disease resected. ${ }^{40,41}$ Therefore, in cases where such microscopic foci of metastatic disease would increase the overall nodal staging based on the number of positive lymph nodes, the clinical implications are less certain. It might be hypothesized that additional positive lymph nodes with a minimal volume of disease may not contribute greatly to an increased risk of relapse. Additional studies will be of benefit in determining the clinical implications of positive lymph nodes that are only detectable by immunohistochemistry. As seminoma is prone to infiltrate as individual cells both in testicular primary tumors and lymph node metastases as found in this study, it is a likely contributor to occult metastasis and detectable by immunohistochemistry; however, it is often amenable to therapy. Application of OCT4 immunohistochemistry in other circumstances, such as nonregional lymph node metastases, may also be of interest to determine a potential role in upstaging of positive cases.

In summary, we concluded that OCT4 immunohistochemistry detects microscopic foci of metastatic seminoma in retroperitoneal lymph node dissection specimens, including the three clinical scenarios of untreated retroperitoneal lymph node dissection with and without clinical suspicion for metastasis and postchemotherapy retroperitoneal lymph node dissection. Untreated retroperitoneal lymph node dissection with clinical suspicion accounted for the most total and proportional additional positive lymph nodes identified by immunohistochemistry. Patterns of occult metastasis included small clusters of cells and single seminoma cells, admixed with sinus histiocytes or centrally within the lymph node parenchyma. Occult foci of embryonal carcinoma were not detected. Further study will be useful to determine whether increased numbers of positive lymph nodes detected by this method with only a small overall volume of metastatic disease contribute to a poorer prognosis after retroperitoneal lymph node dissection than those without immunohistochemically identified small clusters of metastatic tumor cells.

\section{Disclosure/conflict of interest}

The authors declare no conflict of interest.

\section{References}

1 Jones TD, Ulbright TM, Eble JN, et al. OCT4 staining in testicular tumors: a sensitive and specific marker for seminoma and embryonal carcinoma. Am J Surg Pathol 2004;28:935-940.

2 Jones TD, Ulbright TM, Eble JN, et al. OCT4: A sensitive and specific biomarker for intratubular germ cell neoplasia of the testis. Clin Cancer Res 2004;10:8544-8547.

3 Atlasi Y, Mowla SJ, Ziaee SA, et al. OCT4 spliced variants are differentially expressed in human pluripotent and nonpluripotent cells. Stem Cells 2008;26:3068-3074.

4 Gazouli M, Roubelakis MG, Theodoropoulos GE, et al. OCT4 spliced variant OCT4B1 is expressed in human colorectal cancer. Mol Carcinog 2012;51:165-173.

5 Zuk PA. The intracellular distribution of the ES cell totipotent markers OCT4 and Sox2 in adult stem cells differs dramatically according to commercial antibody used. J Cell Biochem 2009;106:867-877.

6 Cheng L, Sung MT, Cossu-Rocca P, et al. OCT4: biological functions and clinical applications as a marker of germ cell neoplasia. J Pathol 2007;211:1-9.

7 Rosner MH, Vigano MA, Ozato K, et al. A POU-domain transcription factor in early stem cells and germ cells of the mammalian embryo. Nature 1990;345:686-692.

8 Hansis C, Grifo JA, Krey LC. Oct-4 expression in inner cell mass and trophectoderm of human blastocysts. Mol Hum Reprod 2000;6:999-1004.

9 Looijenga LH, Stoop H, de Leeuw HP, et al. POU5F1 (OCT3/4) identifies cells with pluripotent potential in human germ cell tumors. Cancer Res 2003;63: 2244-2250.

10 Liu A, Cheng L, Du J, et al. Diagnostic utility of novel stem cell markers SALL4, OCT4, NANOG, SOX2, UTF1, and TCL1 in primary mediastinal germ cell tumors. Am J Surg Pathol 2010;34:697-706.

11 Siegel R, Naishadham D, Jemal A. Cancer statistics, 2012. CA Cancer J Clin 2012;62:10-29.

12 McGuckin MA, Cummings MC, Walsh MD, et al. Occult axillary node metastases in breast cancer: their detection and prognostic significance. Br J Cancer 1996;73:88-95.

13 Scolyer RA, Murali R, McCarthy SW, et al. Pathologic examination of sentinel lymph nodes from melanoma patients. Semin Diagn Pathol 2008;25:100-111.

14 Hubalewska A, Sowa-Staszczak A, Huszno B, et al. Use of Tc99m-nanocolloid for sentinel nodes identification in cervical cancer. Nucl Med Rev Cent East Eur 2003;6:127-130.

15 Nieuwenhuis EJ, van der Waal I, Leemans CR, et al. Histopathologic validation of the sentinel node concept in oral and oropharyngeal squamous cell carcinoma. Head Neck 2005;27:150-158.

16 Murawa D, Nowaczyk P, Hunerbein M, et al. One hundred consecutive cases of sentinel lymph node mapping in colon cancer-the results of prospective, single-centre feasibility study with implementation of immunohistochemical staining. Int J Colorectal Dis 2011;26:897-902.

17 Koljonen V, Bohling T, Virolainen S. Tumor burden of sentinel lymph node metastasis in Merkel cell carcinoma. J Cutan Pathol 2011;38:508-513.

18 Badve S. Sentinel lymph node biopsy, In: Dabbs DJ (ed) Breast Pathology. Elsevier/Saunders: Philadelphia, PA, USA; 2012, pp 93-116.

19 Veronesi U, Paganelli G, Viale G, et al. A randomized comparison of sentinel-node biopsy with routine axillary dissection in breast cancer. $\mathrm{N}$ Engl J Med 2003;349:546-553. 
20 Krag DN, Julian TB, Harlow SP, et al. NSABP-32: Phase III, randomized trial comparing axillary resection with sentinal lymph node dissection: a description of the trial. Ann Surg Oncol 2004;11:208S-210SS.

21 Mansel RE, Fallowfield L, Kissin M, et al. Randomized multicenter trial of sentinel node biopsy versus standard axillary treatment in operable breast cancer: the ALMANAC Trial. J Natl Cancer Inst 2006;98: 599-609.

22 Purushotham AD, Upponi S, Klevesath MB, et al. Morbidity after sentinel lymph node biopsy in primary breast cancer: results from a randomized controlled trial. J Clin Oncol 2005;23:4312-4321.

23 Weaver DL, Krag DN, Ashikaga T, et al. Pathologic analysis of sentinel and nonsentinel lymph nodes in breast carcinoma: a multicenter study. Cancer 2000;88:1099-1107.

24 Weaver DL, Ashikaga T, Krag DN, et al. Effect of occult metastases on survival in node-negative breast cancer. N Engl J Med 2011;364:412-421.

25 Dowlatshahi K, Fan M, Snider HC, et al. Lymph node micrometastases from breast carcinoma: reviewing the dilemma. Cancer 1997;80:1188-1197.

26 Chagpar A, Middleton LP, Sahin AA, et al. Clinical outcome of patients with lymph node-negative breast carcinoma who have sentinel lymph node micrometastases detected by immunohistochemistry. Cancer 2005;103:1581-1586.

27 Cserni G, Bianchi S, Vezzosi V, et al. The value of cytokeratin immunohistochemistry in the evaluation of axillary sentinel lymph nodes in patients with lobular breast carcinoma. J Clin Pathol 2006;59: 518-522.

28 Linden MD, Zarbo RJ. Cytokeratin immunostaining patterns of benign, reactive lymph nodes: applications for the evaluation of sentinel lymph node specimen. Appl Immunohistochem Mol Morphol 2001;9: 297-301.

29 Byrne J, Waldron R, McAvinchey D, et al. The use of monoclonal antibodies for the histopathological detection of mammary axillary micrometastases. Eur J Surg Oncol 1987;13:409-411.

$30 \mathrm{Xu} \mathrm{X}$, Roberts SA, Pasha TL, et al. Undesirable cytokeratin immunoreactivity of native nonepithelial cells in sentinel lymph nodes from patients with breast carcinoma. Arch Pathol Lab Med 2000;124: 1310-1313.

31 Khanna R, Bhadani S, Khanna S, et al. Comparison of immunohistochemistry with conventional histopathology for evaluation of sentinel lymph node in breast cancer. Indian J Surg 2011;73:107-110.

32 Vilardell F, Novell A, Martin J, et al. Importance of assessing CK19 immunostaining in core biopsies in patients subjected to sentinel node study by OSNA. Virchows Arch 2012;460:569-575.

33 Nahrig JM, Richter T, Kuhn W, et al. Intraoperative examination of sentinel lymph nodes by ultrarapid immunohistochemistry. Breast J 2003;9:277-281.

34 Karsten U, Stosiek P. Fast and sensitive immunodetection of carcinoma cells in sentinel nodes. Virchows Arch 2002;440:325-329.

35 Salem AA, Douglas-Jones AG, Sweetland HM, et al. Evaluation of axillary lymph nodes using touch imprint cytology and immunohistochemistry. Br J Surg 2002;89:1386-1389.

36 Lee IK, Lee HD, Jeong J, et al. Intraoperative examination of sentinel lymph nodes by immunohistochemical staining in patients with breast cancer. Eur J Surg Oncol 2006;32:405-409.

37 Abdul-Rasool S, Kidson SH, Panieri E, et al. An evaluation of molecular markers for improved detection of breast cancer metastases in sentinel nodes. J Clin Pathol 2006;59:289-297.

38 Clevenger JA, Kum JB, Alexander RE, et al. Paraganglion cells stain positively for OCT4: a potential pitfall in assessing retroperitoneal lymph node dissection specimens for germ cell tumour metastasis. Histopathology 2013;62:360-363.

39 Ulbright TM. Gonadal teratomas: a review and speculation. Adv Anat Pathol 2004;11:10-23.

40 Albers P, Albrecht W, Algaba F, et al. EAU guidelines on testicular cancer: 2011 update. Eur Urol 2011;60: 304-319.

41 Donohue JP, Thornhill JA, Foster RS, et al. Clinical stage B non-seminomatous germ cell testis cancer: the Indiana University experience (1965-1989) using routine primary retroperitoneal lymph node dissection. Eur J Cancer 1995;31A:1599-1604. 